\title{
Editorial to Multidimensional and Hyphenated Techniques in Separation Science
}

\author{
Jelle De Vos ${ }^{1} \cdot$ Sebastiaan Eeltink ${ }^{1}$
}

Published online: 13 July 2021

๑) The Author(s), under exclusive licence to Springer-Verlag GmbH Germany, part of Springer Nature 2021

The modern challenges that analytical chemists are currently facing demand to improve the number of separated compounds, while also increasing sample throughput. This is a direct consequence of the increased multidisciplinary research endeavors, such as those encountered in the omics field and biomedical applications. Another driving factor is that many modern chemical companies have adopted biotechnology and life sciences as a viable business segment. This has allowed the development of analytical methods, with enhanced separation performance and improved detection sensitivity, at a rapid pace. A recent triumph of modern state-of-the-art separation science can certainly be attributed to the record-breaking development of biotechnological vaccines for SARS-CoV-2. However, for complex analytical problems, still many challenges lay ahead.

This Topical Collection focuses on recent advances in Multidimensional and Hyphenated Techniques in Separation Science. The latest highlights in using multidimensional
LC technology for chiral separations are highlighted by Ali and coworkers. Davis provides a theoretical treatment, using statistical overlap theory, applied to small second-dimension peak capacity. Striegel and Trainoff demonstrate the power of size-exclusion chromatography hyphenated to online viscosimetry and refractive index detection for the separation of a diastereomeric pair of monosaccharides. It goes without saying that the excellent work by these researchers shows the innovations that were recently realized to advance separation science, and our sincere gratitude goes out to all authors and reviewers that made this Topical Collection possible.

Guest Editors,

Dr. Jelle De Vos, Vrije Universiteit Brussel

Prof. Dr. Sebastiaan Eeltink, Vrije Universiteit Brussel

Publisher's Note Springer Nature remains neutral with regard to jurisdictional claims in published maps and institutional affiliations.
Jelle De Vos

Jelle.De.Vos@vub.be

1 Vrije Universiteit Brussel, Elsene, Belgium 